\title{
The Lithology Discrimination with Back-Propagation Neural Network
}

\section{Method}

\author{
Liu ShaoHua(1,2), a , Duan XiaoQiu ${ }^{(1,2), b}$, Wang ZhongHao ${ }^{(1,2), c}$, And Wu \\ Dong $^{3, d}$
}

${ }^{1}$ Key Laboratory of Exploration Technologies for Oil and Gas Resource(Yangtze University), Ministry of Education,Jingzhou,Hubei,434023, China

${ }^{2}$ School of Geoscience of Yangtze University WuHan,Hubei,430100,China

${ }^{3}$ CNPC Greatwall Drilling Company ,Beijing,102200, China

a Ish811@126.com, b893401147@qq.com , '71306009@qq.com, d403192095@qq.com

Keywords: logging data, lithology identification, Back-Propagation Algorithm (BP) neural network

Abstract: In lithology identification method, artificial neural network recognition results du-e to its objective and reliable, to be more widely used. Study selection of BP neural net-work, Shen 630-H1426 logging lithology identification, prediction accuracy of 90\%, with a higher prediction accuracy magmatic rocks and metamorphic crocks. Through analysis sho-ws that BP neural network to predict lithology is a more reliable method.

\section{Introduction}

Reservoir prediction is one of the most basic jobs to find oil and gas resources, oil and gas reserves evaluation. Due to the underground geological structure complexity and logging parameters are fuzzy, the traditional lithology identification methods are precision co..

The method of artificial neural network based on their unique ability to obtain a sample of learning pattern recognition, to overcome the defect of the traditional method of lithology identification. It is self-organizing, self-learning, adaptive, fault tolerance and anti-interference ability, the recognition result is objective and reliable, using this method to identify lithology logging lithological identification work is improved.

Application of neural network in the field of well logging lithological identification, the predecessors have done a lot of research. Lu Xinwei, Jin Zhangdong, the lithology of Sheng-li oil field by using BP neural network to identify [1], and Fan Xunli BP network of Tarim OilfieldTZ4 well logging lithology automatic identification[2]. Hou Junsheng, Wang Ying, use-d the BP network to identify the quantitative interpretation of on gas logging data[3], the reco-gnition accuracy rate is very high. However, did not relate to the important problem of the convergence properties of BP algorithm.

\section{Back-Propagation(BP) neural network}

Basic principles

Artificial neural network model[4] has dozens of kinds, the typical BP network and Kohen network are widely used in petroleum industry, and has achieved very good results 
in logging interpretation, seismic data processing. The purpose of this study is to use natural potential, natural gamma curve, the curve of acoustic logging, resistivity curve to forecast the rock [5], so they use BP neural network. The network proposed by Rumethart and someone else in 1985, is a kind of feed-forward network, also known as the error back propagation network. The basic neural network consists of an input layer, one hidden layer and output layer consisting of a three layer network, as shown in Figure 1.

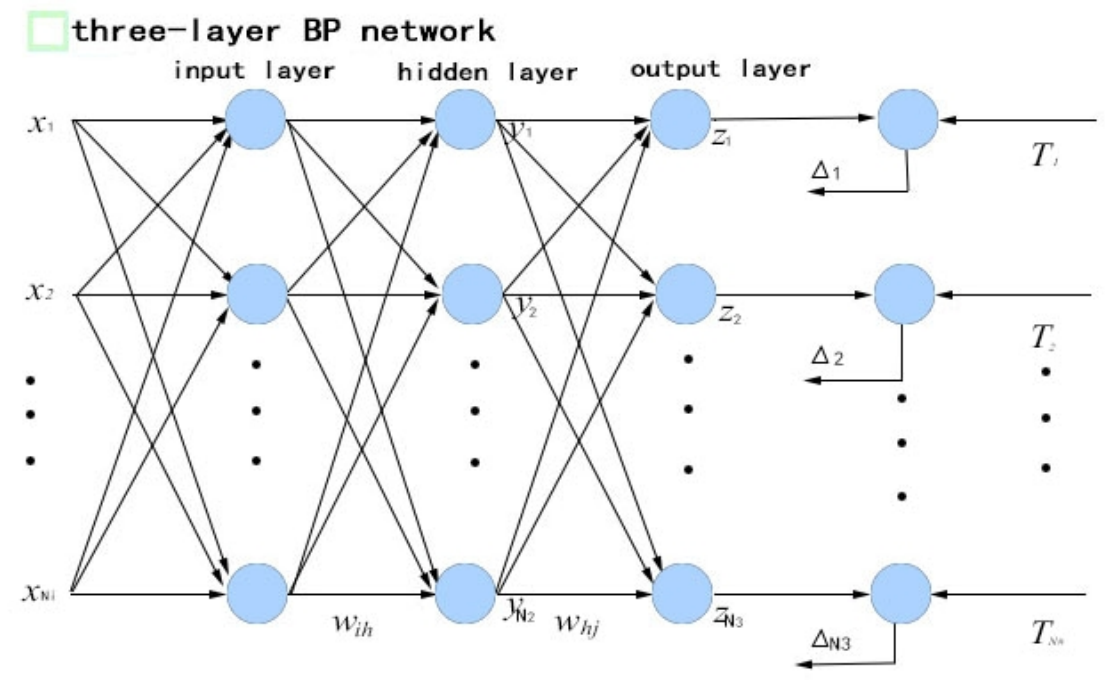

Fig.1 Three - layer neural network topology diagram and calculations

\section{Basic steps}

1) Initialization: initialize the network learning rate (L), weight (W) and bias (theta) learning parameters.

2) Lithological data normalization:

$$
\Delta \text { Lith }=\frac{\text { Lith }- \text { Lith }_{\min }}{\text { Lith }_{\max }-\text { Lith }_{\min }}
$$

3) Forward propagation to calculate the output layer neurons (Fig. 2):

Output: $\mathrm{I}_{\mathrm{j}}=\Sigma \mathrm{iW} \mathrm{W}_{\mathrm{ij}} \mathrm{X}_{\mathrm{i}}+\theta_{\mathrm{j}} \quad \mathrm{X}_{\mathrm{j}}=\mathrm{f}\left(\mathrm{I}_{\mathrm{j}}\right)=1 /\left(1+\mathrm{e}-\mathrm{I}_{\mathrm{j}}\right)$

4) Back-propagation calculation error (Fig.3):

Calculation error of the output layer nodes: Errj=Oj(1-O $\left.\mathrm{O}_{\mathrm{j}}\right)\left(\mathrm{T}_{\mathrm{j}}-\mathrm{O}_{\mathrm{j}}\right) \quad$ (Including: $\mathrm{O}_{\mathrm{j}}=\mathrm{X}_{\mathrm{j}}, \mathrm{T}_{\mathrm{j}}$ as the actual output) Hidden layer node $J$ errors :Errj $=\mathrm{O}_{\mathrm{j}}\left(1-\mathrm{O}_{\mathrm{j}}\right) \Sigma \mathrm{k} \quad \operatorname{Err}_{\mathrm{k}} \mathrm{W}_{\mathrm{jk}}$ (Including, $\mathrm{Wjk}$ are the weights of the nodes to the node, Errk is the error of node $\mathrm{K}$ )

Weight updating formula: $\Delta \mathrm{W}_{\mathrm{ij}}=(\mathrm{l}) \mathrm{Err}_{\mathrm{j}} \mathrm{O}_{\mathrm{i}}$, so $\mathrm{W}_{\mathrm{ij}}=\mathrm{W}_{\mathrm{ij}}+\Delta \mathrm{W}_{\mathrm{ij}}$

Bias updating formula: $\Delta \theta_{\mathrm{j}}=(\mathrm{l}) \operatorname{Err}_{\mathrm{j}}$, so $\theta_{\mathrm{j}}=\theta_{\mathrm{j}}+\Delta \theta_{\mathrm{j}}$

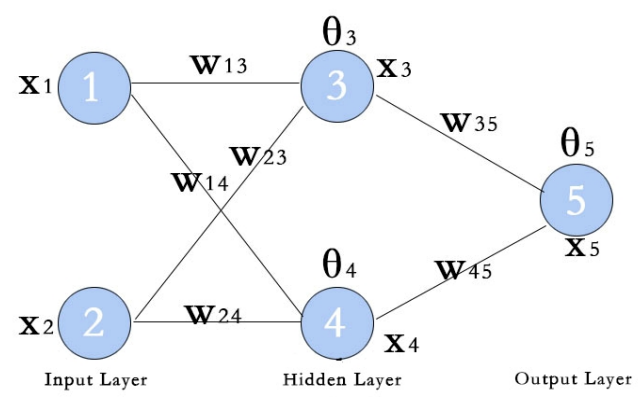

Fig.2 Forward propagation diagram

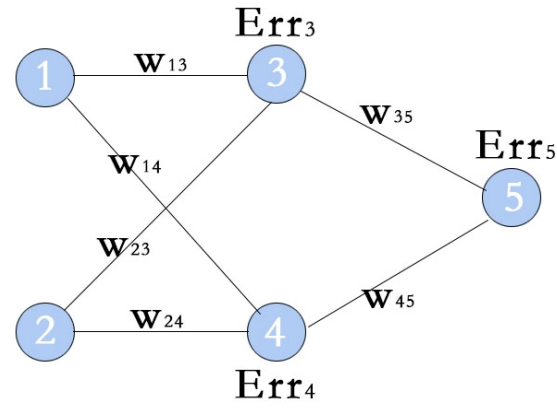

Fig.3 Back - propagation diagram 


\section{The algorithms of data}

BP neural network is to calculate the output, based on back propagation calculation error, the final iteration to adjust the weights and bias.

\section{Learning parameter class:}

public double_LearnRate;//Learning rate;

public int_InSum;//Input layer node number;

public int_OutSum;//Output layer node number;

public double_Momentum; //Learning step;

public int_LearnTimes; //Learning number;

public double_LearnDouble;//Learning error;

public int_Times;

public double_ErrorValue; //error

public String_SampleFileName;//name;

Data storage class:

public ArrayList $<$ Nodes $>$ _BPmodel=new ArrayList $<$ Nodes $>()$; //bp model;

public float[][] _InputDataSet;//Sample Data;

public int_InputDataSetRows;//row;

public int_InputDataSetCols;//column;

public double_ExpectOut;//Desired output

public double_SqrtErr;//error

\section{BP Node class:}

public ArrayList $<$ Node $>$ _Nodes $=$ new ArrayList $<$ Node $>() ; / /$ Node

Node class:

public double Threshold;//bias

public double DThreshold; / the change of the bias value

public ArrayList $<$ Double $>$ Weight $=$ new ArrayList $<$ Double $>() ; / /$ weight

\section{Example of application}

The system combined with JavaSE, java drawing technology, by using the knowledge ofdesign of swing framework and the BP neural network theory and. The use of NetBeans dev-elopment tools, the use of integrated lithology recognition system based on Java language de-velopment.

In the process of system implementation, firstly, Shen 630-H1426 logging data process-ing, data storage model; the stored data to select the appropriate curve and using a comb-ination method of BP neural network to realize the recognition of lithology. 


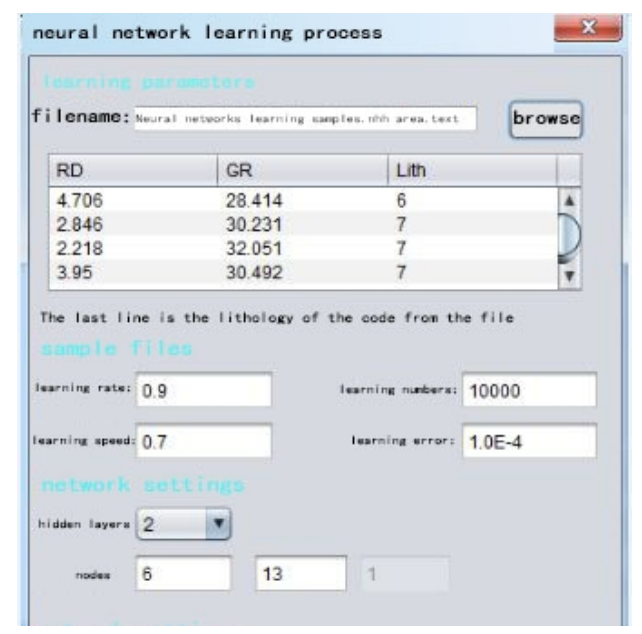

Fig.4 The main interface of neural network

\begin{tabular}{lll}
\hline RD GR & Lith & \\
4.706 & 28.414 & 6 \\
2.846 & 30.231 & 7 \\
2.218 & 32.051 & 7 \\
3.95 & 30.492 & 7 \\
1.47 & 18.934 & 7 \\
5.374 & 18.952 & 6 \\
2.63 & 15.864 & 6 \\
3.624 & 23.624 & 6 \\
2.327 & 35 & 7 \\
8.16 & 23.054 & 6
\end{tabular}

Fig.5 Sample data

\section{Conclusion}

Research shows that for well logging lithological identification based on BP neural network, the method is simple and easy to operate, and the recognition accuracy rate is very high. This provides a new method for geological interpretation of logging data, the accuracy of search and identification of oil and gas formation, in the field of oil and gas resources development has a very practical significance [6].

\section{Acknowledgements}

This work was financially supported by the Education Department of Hubei province science and technology research projects(D20141301), also supported by the development of science and technology project of China National Petroleum Corporation(2013E-3809).

\section{References}

[1]Lu XinWei, Jin ZhangDong. The lithology identification method of feed forward neural networks. Oil and gas geology,1993

[2]Fan XunLi, Dai Hang, Zhang XinJia,etc. The application of neural network to the lithology identification. Logging technology,1991,1.

[3]Hou JunSheng, Wang Ying. Neural network method in the application of gas layer logging data interpretation.Geological and prospecting,199,5.

[4]Sun MingGuang, Zhang YunLian. New type PDC bit design.2000,22(2):31-34.

[5]Li GongQuan, Cao DaiYong, Chen GongYang,etc. Under the condition of PDC bit drilling lithology identification. Oil drilling process. 2006,4(28):2.

[6]Zhang Hong, Chu LeJun, Shen XiaoHua. The Back-Propagation neural network application to lithological identification. Geological prospecting.2002,11(38):6 